\title{
Optimization of Pump Hydraulic Performance Based on the Response Surface Method
}

\author{
Shengli Xu1 ${ }^{*}$, Shaowei Zhong1, Haixin Zhao ${ }^{2}$, Xin Peng1 \\ 1 School of energy and power engineering, Dalian university of technology, Liaoning, China; \\ weizs@mail.dlut.edu.cn(S.H.Z.); pengxin@mail.dlut.edu.cn(X.P.) \\ 2 Department of engineering mechanics, Dalian university of technology, Liaoning, China; \\ zhxstc@mail.dlut.edu.cn(H.X.Z.) \\ * Correspondence: xusl@dlut.edu.cn; Tel.: +86-138-9864-8586
}

\begin{abstract}
This paper studies the optimization method of pump hydraulic performance based on the response surface method. A parametric model of impeller and diffuser is established. Three-dimensional optimization is carried out on the basis of the initial model obtained by one-dimensional design method. We select the pump hydraulic efficiency and the head as objective function and constraint function. Response surface models are constructed to analyze the relationship between the objectives and the design variables, and the global optimization of hydraulic performance is realized. According to the internal flow characteristics of pump, this paper proposes the strategy of two steps optimization, which aims at meridional plane and blade shape, respectively, to solve the problem of large numbers of design parameters and computational cost. The optimization results show that the hydraulic efficiency of pump increased by $3.7 \%$, and the head is nearly the same.
\end{abstract}

Keywords: pump hydraulic performance; optimization design; response surface method; parameterization; meridional plane

\section{Introduction}

In recent years, CFD technology is widely used in numerical calculation of three-dimensional viscous flow field of fluid machinery. At the same time, CFD numerical calculation is no longer limited to studying the internal flow field of pump, but used to improve the efficiency of pump cooperating with the optimization algorithm. Skerlavaj, et al[1], set up various response surface models for design parameters and performance functions. The MOGA-II algorithm was used to optimize the hydraulic performance of single stage double suction centrifugal pumps. The results show that the radial basis function has high accuracy and computational efficiency. Huang [2] combined with radial basis response model and NSGA-II genetic algorithm to optimize the impeller of mixed flow pump. The hydraulic efficiency increased by $1.2 \%$ with head increased by $0.5 \mathrm{~m}$. Yuan [3] combined the kriging surrogate model with multi-island genetic algorithm to optimize the impeller shape of a low specific speed centrifugal pump for improving its performance. This method, by establishing the response relation for performance functions and combining with optimization algorithm, is widely applied in the practical engineering structural optimization and especially suitable for calculating the time-consuming nonlinear optimization problems [4-6]. Among them, CORSRBF algorithm has better effect in dealing with optimization problems of nonlinear constraints [7]. However, all the researches mentioned above were limited to the impeller optimization design. The literatures about optimal design concerning the impeller and diffuser together were rare [8-9]. However, fluid machinery is complex, and the best performance of impeller can't guarantee the best of whole pump. It is necessary to consider both the impeller optimization and the diffuser optimization to obtain better hydraulic performance of pump. Kim [10] used the experimental design method to analyze the influence of design parameters of impeller and diffuser on the efficiency and head of mixed flow pump. A response surface model for hydraulic optimization was constructed to optimize the performance. The results show that the design parameters at the inlet of diffuser directly affect the fluid flow at the exit of impeller, and the pump efficiency increased by $2.67 \%$. Therefore, it is meaningful to consider the hydraulic optimization of meridional channel and blade shape of impeller and diffuser at the same time.

The parameterized model is the prerequisite for the optimal design of pump hydraulic performance. How to use the parametric curve to express the blade geometry has always been the focus of study. Casey [11] defined the impeller meridional channel through the Bezier polynomial, but the number of its control points was too much and it was difficult to easily change the shape of blade profiles. Braembussche, et al [12], applied the fourth-order Bezier 
curve to describe the profiles of shroud and hub, which realized parameterization of impeller profiles with fewer control parameters. The method is also widely used in geometrical parameterization of rotating mechanical structures.

This paper proposes a meridional plane parameterization method of impeller and diffuser by Bezier polynomial. Blade three-dimensional shape is controlled in combination with a definition of blade camber line by the distribution of blade angles. Then the parametric model of pump is realized by the complete structural characteristic parameters. The pump efficiency and head are calculated through flow field analysis using the software CFX. Surrogate models are constructed to analyze the relationship between the objectives and the design variables. The CORS constraint optimization algorithm is applied to solve the optimization problem to determine the best combination of optimization parameters. Finally, the internal flow characteristic is then deeply analyzed to illustrate the performance improvement of pump.

\section{Pump Geometric Parameterization Method}

Pump impeller and diffuser are mainly composed of impeller blades and diffuser blades. The meridional plane of blade is closely related to the hydraulic performance and structural reliability of pump. The fourth-order Bezier curves are introduced into the description of meridional profiles at hub and shroud for the less control points and parameters, which is conducive to the parametric design of blade. The meridional profiles at hub and shroud are made up of four Bezier curves. Each curve endpoints are determined by the basic design parameters (Inlet and outlet diameter of impeller, trailing edge width of impeller, outlet diameter and trailing edge width of diffuser). The slope of curve endpoints is defined, and intermediate control points move in the tangential direction of two endpoints of the curve. This parameterization method not only controls the direction of fluid flowing into and out of the impeller, but also controls the shape of the meridional profiles at hub and shroud with a small number of control points. In order to guarantee the slope continuity of these profiles between the impeller and diffuser, the slope at the ending points of impeller profiles equals the one at the starting points of diffuser profiles. The meridional contours between impeller and diffuser are linked through the impeller-diffuser gap. The meridional parameterization model is shown in Figure 1.

Table 1 shows the parameterization mode on the meridional profiles at the hub of impeller and diffuser. The parameterization method at the shroud is the same as the one at the hub. The points $\mathrm{P}_{2}$ and $\mathrm{P}_{10}$ move along the meridional profiles at shroud and hub, respectively, to determine the position of the blade leading edge.

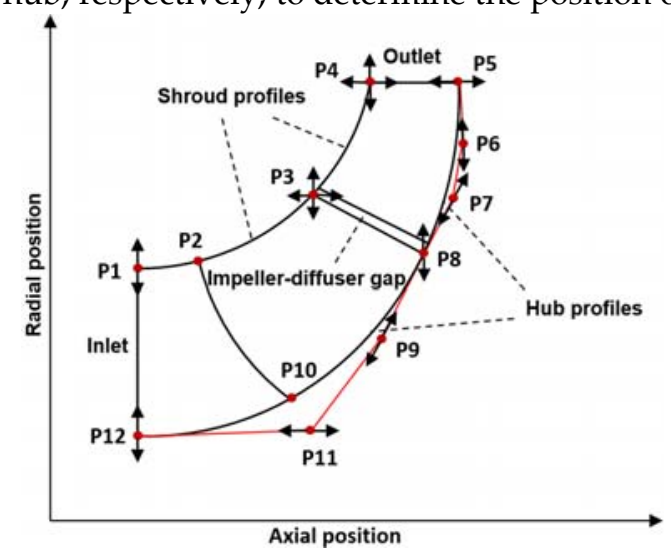

Figure 1. Meridional plane defined by Bezier curves 
Table 1. Parameterization mode on the meridional profiles at the hub

\begin{tabular}{|c|c|c|c|}
\hline Meridional profile at the hub & Control point & Control mode & Control parameters \\
\hline \multirow{4}{*}{$\begin{array}{l}\text { Meridional profile at the hub } \\
\text { of impeller }\end{array}$} & $\mathrm{P}_{12}$ & Move up and down & $d_{h}$ \\
\hline & $\mathrm{P}_{11}$ & $\begin{array}{l}\text { Moves along the tangential } \\
\text { direction of the leading point } \mathrm{P}_{12}\end{array}$ & $h \_k 1 、 h \_x 1$ \\
\hline & $\mathrm{P}_{9}$ & $\begin{array}{l}\text { Moves along the tangential } \\
\text { direction of the trailing point } \mathrm{P}_{8}\end{array}$ & $h \_k 2 、 h \_x 2$ \\
\hline & $\mathrm{P}_{8}$ & Move up and down & h_d2 \\
\hline \multirow{4}{*}{$\begin{array}{l}\text { Meridional profile at the hub } \\
\text { of diffuser }\end{array}$} & $\mathrm{P}_{8}$ & Move up and down & h_d2 \\
\hline & $\mathrm{P}_{7}$ & $\begin{array}{l}\text { Moves along the tangential } \\
\text { direction of the leading point } \mathrm{P}_{8}\end{array}$ & h_k2、h_x3 \\
\hline & $\mathrm{P}_{6}$ & $\begin{array}{l}\text { Moves along the tangential } \\
\text { direction of the trailing point } P_{5}\end{array}$ & h_k3、h_x4 \\
\hline & $\mathrm{P}_{5}$ & Move around left and right & $b_{3}$ \\
\hline
\end{tabular}

Blade angles are the basic parameters that control the blade shape. Blade angles at inlet and outlet are selected as the control parameters, which determined the wrap angles. Figure 2 shows the blade angle distribution on the meridional profiles. Where the abscissa is the relative length of meridional profiles, and the ordinate is the blade angle. Blade can be given a suitable blade angle distribution by moving the endpoints of the curve. The length of meridional profiles is $\mathrm{L}$, so the relative length is defined as $l_{r e l}=\mathrm{l}(\mathrm{u}) / \mathrm{L}$, which changes from 0 to 1 . Finally, parametrized fluid model of impeller and diffuser is shown as Figure 3.

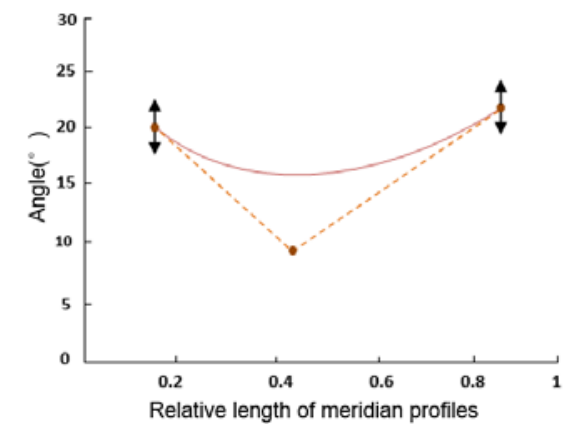

Figure 2. Blade angle distribution on the meridional profiles

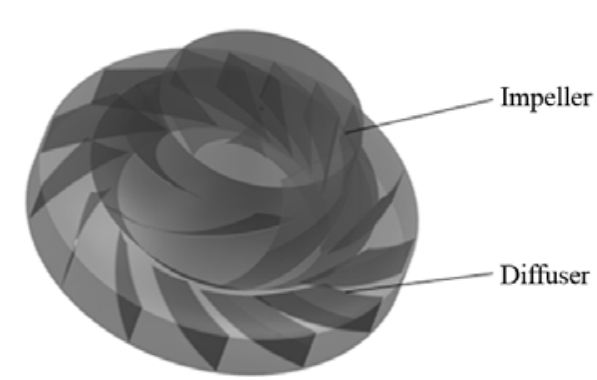

Figure 3. Parametrized fluid model for impeller and diffuser

\section{Mathematical Formula and Solving Methods for Pump Performance Optimization}

\subsection{Mathematical Formula of Optimization Problem}

The optimization objective is pump hydraulic efficiency, and pump head is selected as a constraint to limit the pump input power. Considering the large number of design variables of whole pump, this paper proposed two-step optimization for the meridional plane and blade shape, respectively. The difference between the two-step optimization formulations is only optimization parameters.

For the first optimization aiming to the meridional plane, we select the shape characteristic parameters of the meridional plane of impeller and diffuser as the optimization parameters. The optimization formulation is shown below.

$$
\begin{aligned}
& \text { Find } x=\left(d_{h}, g a p, d_{2}, b_{3}, d_{3}, s_{-} k 2, h \_k 2, s_{-} k 3, h_{-} k 3\right) \\
& \text { Max } f(x, p) \\
& \text { s.t } \quad h^{L} \leq h(x, p) \leq h^{U} \\
& \quad x_{i}^{L} \leq x_{i} \leq x_{i}^{U} \quad i=1,2, \ldots, 9
\end{aligned}
$$

where $\mathrm{f}(\boldsymbol{x}, \boldsymbol{p})$ and $\mathrm{h}(\boldsymbol{x}, \boldsymbol{p})$ represent the surrogate models of hydraulic efficiency and pump head, respectively. $h^{L}$ and 
$h^{U}$ are the upper and lower limits of head. $\boldsymbol{p}$ is a fixed parameter vector. $x_{i}$ is the optimized parameters. $x_{i}^{U}$ and $x_{i}^{L}$ represent the upper and lower limits of $x_{i}$. Among the optimization parameters, $d_{h}$ is the impeller hub diameter. gap is the impeller-diffuser clearance. $d_{2}$ is the impeller outlet diameter. $b_{3}$ is the exit edge width of diffuser. $s_{-} k 2$ and $h \_k 2$ are the slope at the end of meridional profiles of shroud and hub, respectively. Similarly, $s_{-} k 3$ and $h \_k 3$ are the starting slope of the profiles of shroud and hub, respectively.

For the second optimization aiming to the blade shape, we select the inlet and outlet blade angles of impeller and diffuser as the optimization parameters. This optimization formulation is shown below.

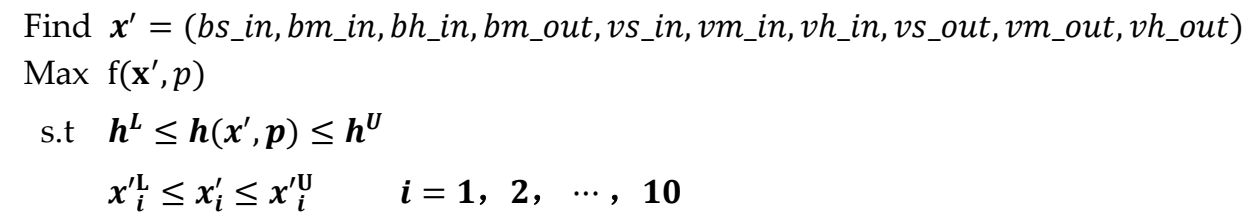

where $b s_{-} i n, b m_{-} i n$ and $b h_{-} i n$ are the inlet blade angle of impeller shroud profile, intermediate profile and hub profile, respectively. bm_out is the outlet blade angle of impeller intermediate profile. The optimization parameters of diffuser are the same as those of impeller.

\subsection{Global Optimization}

Automatic optimization process includes the parameterized model update, model meshing, flow field calculation, and extracting computational objective function and constraint function values. We used the radial basis function (RBF) to approximate the hydraulic efficiency and pump head with optimization parameters. CORS-RBF algorithm was adopted to optimize the response surface model to realize the automatic optimization of pump hydraulic performance. The flowchart of optimization process is shown as Figure 4.

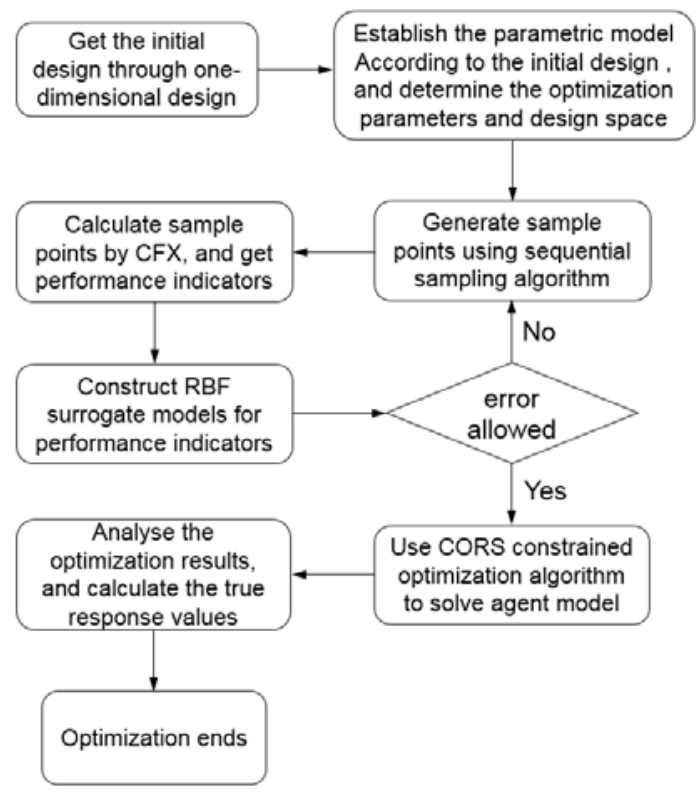

Figure 4. Automatic optimization process based on RBF 


\section{Optimization Example}

The mixed-flow pump of high specific speed, whose casing was specified as the kind of special spherical shape, was taken to be optimized according to the internal flow field analysis and external characteristics. The design head is $17.6 \mathrm{~m}$. Mass flow rate is $384.7 \mathrm{~m}^{3} / \mathrm{h}$. Rotating speed is $1850 \mathrm{rpm}$.

\subsection{The Settings of Calculation Model}

Numerical analysis was carried out using a commercial computational fluid dynamic software CFX. The single passages with impeller import extension section, one impeller passage and one diffuser passage were simulated. The single passage model can contribute to the reduction of numerical simulation's source and time. The whole fluid calculation model is shown in Figure 5. The standard $k-\omega$ turbulence model was selected to close the governing equation. Static pressure was set at the inlet plane with mass flow rate at the outlet plane of volute. All solid walls were applied non-slip boundary conditions. Rotational periodicity conditions were set on both sides of single passages. Frozen rotor interface mode was used between the impeller and diffuser, and stage-average interface mode was used between the diffuser and volute.

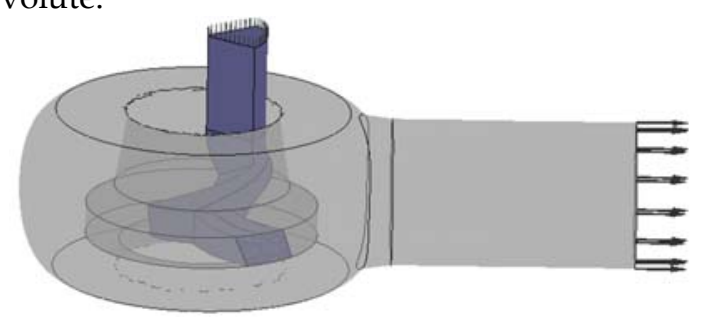

Figure 5. Pump calculation model of single passages

The computational domains were discretized using unstructured grids. The interfaces and blade walls were meshed with smaller grids to ensure the accuracy of calculation results. Thickness of the first boundary layer of blade is $0.05 \mathrm{~mm}$ and that of volute is $0.1 \mathrm{~mm}$. The largest value of Yplus is 67 which meets the Yplus requirement of the turbulence model. In addition, before conducting the numerical simulation, it is important to consider the number of grids, especially for the pump optimization process. Grid-independent validation was checked using five groups of grids. As shown in Figure 6, the hydraulic efficiency and pump head change slowly as the number of grids is greater than 1.46 million. As a result, 1.5 million grids were used to define the whole computational domain. The grid calculation model is shown in Figure 7.

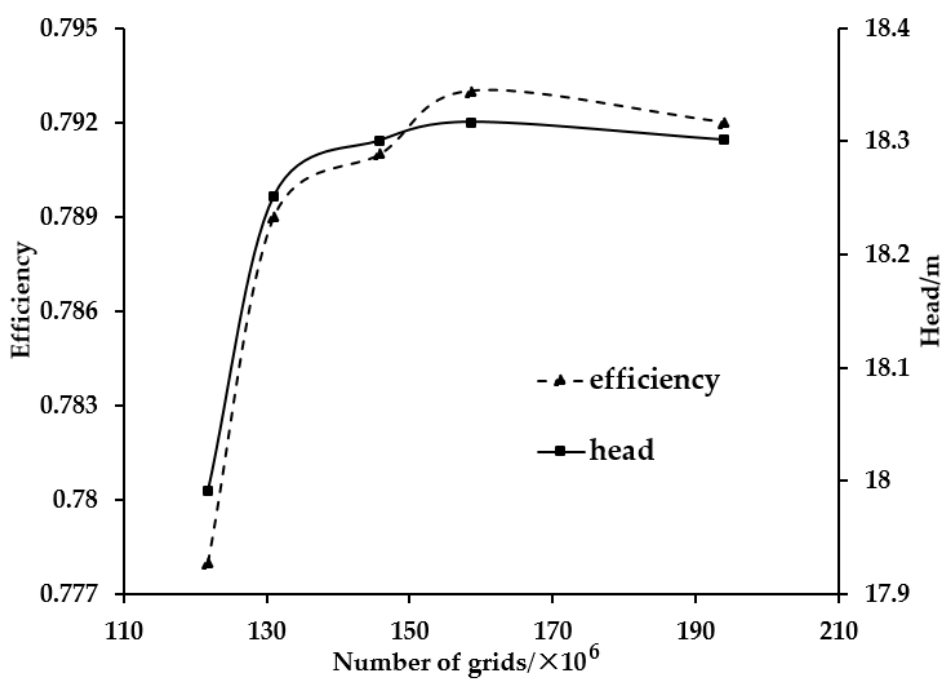

Figure 6. Results of grid-independent validation 


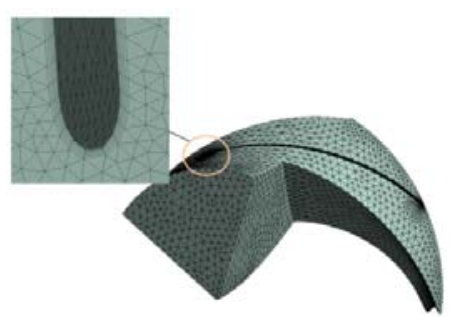

(a)

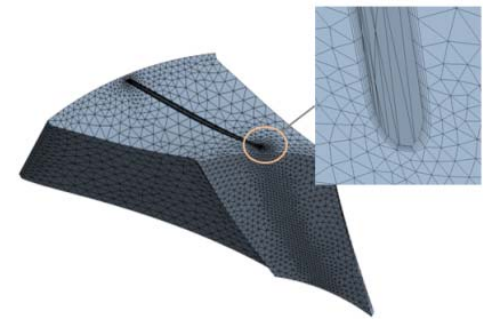

(b)

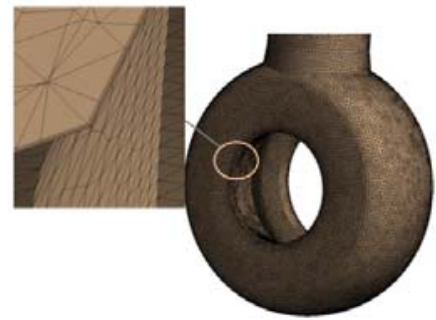

(c)

Figure 7. Grids of fluid domain and boundary layer: (a)The single passage of impeller; (b) The single passage of diffuser; (c) Volute

\subsection{Optimization Results}

In the first step optimization of the meridional plane, surrogate model was constructed by radial basis function with 42 design sample points specified in the design space. Table 2 shows parameters optimization results of the meridional plane. Based on the first optimization, the blade shape optimization was carried out. Ten parameters of the second blade shape optimization specified 45 sample points and parameters optimization results are shown in Table 3.

Table 2. Parameters optimization results of the meridional plane

\begin{tabular}{cccc}
\hline Optimization parameters & Initial size $/(\mathbf{m m})$ & Design space/(mm) & Optimization results /(mm) \\
\hline$d_{h}$ & 116 & {$[100,120]$} & 116 \\
gap & 6 & {$[2,9]$} & 7.4 \\
$d_{2}$ & 157 & {$[155,160]$} & 160 \\
$b_{3}$ & 78 & {$[68,88]$} & 71 \\
$d_{3}$ & 488 & {$[480,508]$} & 480 \\
$s_{-} k 2$ & 1 & {$[0.7,1.4]$} & 1.4 \\
$h \_k 2$ & 1.18 & {$[1,1.6]$} & 1.6 \\
$s_{-} k 3$ & 6 & {$[3,9]$} & 4 \\
$h \_k 3$ & 5 & {$[4,9]$} & 6.2 \\
\hline
\end{tabular}

Table 3. Parameters optimization results of the blade shape

\begin{tabular}{cccc}
\hline Optimization parameters & Initial size $/(\mathbf{m m})$ & Design space/(mm) & Optimization results /(mm) \\
\hline bs_in & 15 & {$[10,25]$} & 13.6 \\
bm_in & 24 & {$[15,30]$} & 15.0 \\
bh_in & 27 & {$[15,35]$} & 17.5 \\
bm_out & 29 & {$[22,31]$} & 22.6 \\
vs_in & 43 & {$[33,53]$} & 51.4 \\
vm_in & 33 & {$[23,43]$} & 36.3 \\
vh_in & 35 & {$[25,45]$} & 33.9 \\
vs_out & 49 & {$[40,55]$} & 54.7 \\
vm_out & 50 & {$[40,60]$} & 56.1 \\
vh_out & 53 & {$[44,61]$} & 60.8 \\
\hline
\end{tabular}


The hydraulic efficiency of the initial model is $80.3 \%$, and pump head is $18.5 \mathrm{~m}$. The initial model was obtained through one-dimensional design, without more detailed optimization and improvement. After the first optimization, the efficiency and head are $86.4 \%$ and $17.3 \mathrm{~m}$, respectively. Compared with the first optimization, the efficiency of the second optimized pump increased by $0.6 \%$, and the head is $18 \mathrm{~m}$. Optimization accuracy between the predicted values of the surrogate models and the real CFD values for the optimal results was conducted, as shown in Table 4.

Table 4. Comparison between predictive values of RBF surrogate models and CFD analysis

\begin{tabular}{|c|c|c|c|c|c|c|}
\hline \multirow[t]{2}{*}{ Performance } & \multicolumn{2}{|c|}{$\begin{array}{c}\text { The first meridian } \\
\text { optimization }\end{array}$} & \multirow[t]{2}{*}{$\begin{array}{l}\text { Relative error } \\
(\%)\end{array}$} & \multicolumn{2}{|c|}{$\begin{array}{l}\text { The second blade } \\
\text { shape optimization }\end{array}$} & \multirow[t]{2}{*}{$\begin{array}{l}\text { Relative error } \\
(\%)\end{array}$} \\
\hline & $\mathrm{RBF}$ & CFD & & $\mathrm{RBF}$ & CFD & \\
\hline Efficiency (\%) & 85.6 & 86.4 & 0.93 & 87.8 & 87 & 0.92 \\
\hline Head (m) & 16.8 & 17.3 & 2.9 & 17.7 & 18 & 1.6 \\
\hline
\end{tabular}

\subsection{The Internal Flow Field Analysis}

The optimal results were verified using numerical analysis, and the internal and external characteristics for both the optimum model and the initial model were compared to find the reasons for the improved pump performance. In order to reduce the calculation time, the single flow passage was adopted to perform hydraulic performance optimization. CFD calculation. Results of external characteristics between single flow passage and entire flow passage are shown as Table 5. It is obvious that relative errors between the single and entire flow passage can be kept within 3 percent, except for the head error of initial model. The internal instability flow of initial model leads to the greater deviation than the optimal models. The results show that efficiency of single flow passage optimization increased by $6.3 \%$, corresponding to the entire passage increased by $3.7 \%$.

Figure 8 shows velocity distribution of stream surface at the single flow passage and entire flow passage. The inlet of impeller due to the weak work capacity, coupled with the impact loss of the blade leading edge, was prone to the local vortex. After the first meridional plane optimization, the area of the low pressure gradually reduced. The blade shape was optimized so that the blade angles were consistent with the fluid flow angles, and the inlet low pressure zone near the blade leading edge disappeared completely. Since the flow state in the impeller directly affected the flow field distribution of diffuser, the more pronounced changes happened in the flow field of diffuser. From the entire flow passage velocity contour, we can see that the large low velocity whirlpool developed near the suction surface of diffuser, and were distributed in the fixed flow path of diffuser. After the meridian was optimized, the vortex scale became smaller and moved to the diffuser outlet. Finally, the partial vortex of diffuser was also suppressed. Those were the same as the single passage analysis results. Calculation results of single passage can't fully reflect the flow field law of entire passage, but can reveal the optimization direction of flow field. Optimum model has the improved efficiency with the stable flow field compared to the initial model. Relative streamline distribution before and after optimization is shown as Figure 9. The vortex disappeared gradually and vortex intensity weakened after optimization. Streamline distribution in the axial flow channel was more uniform.

Table 5. Comparison of CFD analysis between single flow passage and entire flow passage

\begin{tabular}{cccccccccc}
\hline & \multicolumn{3}{c}{ The initial model } & \multicolumn{2}{c}{ The meridian optimization } & \multicolumn{2}{c}{ The blade shape optimization } \\
\cline { 2 - 11 } Performance & $\begin{array}{c}\text { Single } \\
\text { flow } \\
\text { passage }\end{array}$ & $\begin{array}{c}\text { Entire } \\
\text { flow } \\
\text { passage }\end{array}$ & $\begin{array}{c}\text { Relative } \\
\text { error }(\%)\end{array}$ & $\begin{array}{c}\text { Single } \\
\text { flow } \\
\text { passage }\end{array}$ & $\begin{array}{c}\text { Entire } \\
\text { flow } \\
\text { passage }\end{array}$ & $\begin{array}{c}\text { Relative } \\
\text { error(\%) }\end{array}$ & $\begin{array}{c}\text { Single } \\
\text { flow } \\
\text { passage }\end{array}$ & $\begin{array}{c}\text { Entire } \\
\text { flow } \\
\text { passage }\end{array}$ & $\begin{array}{c}\text { Relative } \\
\text { error(\%) }\end{array}$ \\
Efficiency $(\%)$ & 80.3 & 82.0 & 2.1 & 86.4 & 85.1 & 1.5 & 87.0 & 85.7 & 1.5 \\
Head ( & 18.5 & 19.2 & 3.6 & 17.3 & 17.5 & 1.1 & 18.0 & 18.4 & 2.2 \\
\hline
\end{tabular}




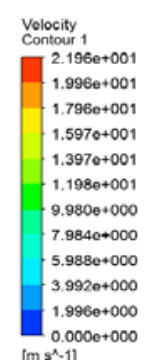

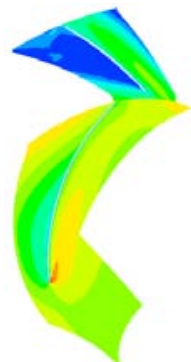

(a)

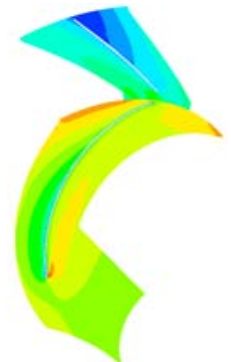

(b)

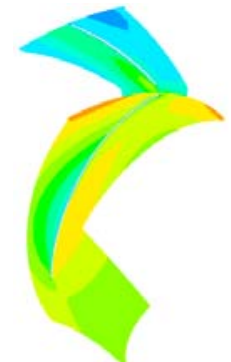

(c)

a. Relative velocity distribution of stream surface at the single flow passage: (a) The initial model; (b) The meridian optimization; (c) The blade shape optimization.

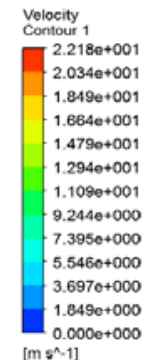

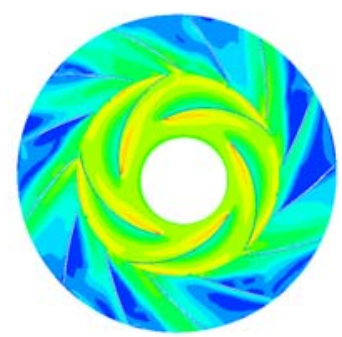

(a)

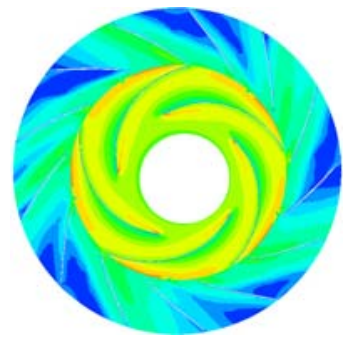

(b)

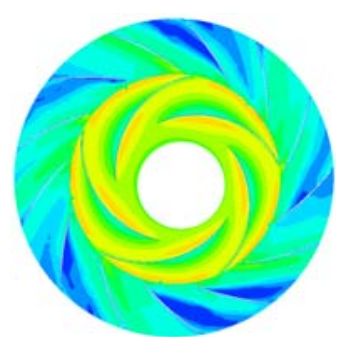

(c)

b. Relative velocity distribution of stream surface at the entire flow passage: (a) The initial model; (b) The meridian optimization; (c) The blade shape optimization.

Figure 8. Relative velocity distribution of stream surface at the middle span height of impeller and diffuser

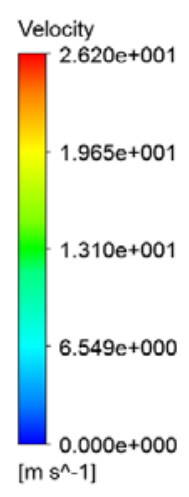

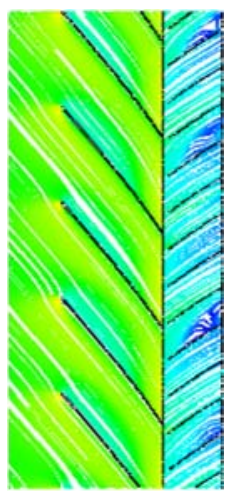

(b)

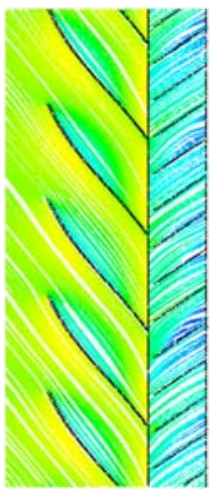

(c)

Figure 9. Comparison of relative streamline distribution at the entire flow passage: (a) The initial model; (b) The meridian optimization; (c) The blade shape optimization

Figure 10 shows the total pressure distribution on the meridional plane of impeller and diffuser. Pressure increased along with the passage. In the case of the initial model, the degree of total pressure rise on the diffuser was clearer than the optimized models. Pressure near the hub side was higher, which lead to nonuniform flow and resulted in the reduction of the hydraulic efficiency. In addition, the difference of pressure distribution between the initial design and the first optimal model was obviously bigger than that between the two optimal models because blade angles had little effect on the performance of meridional channel. 


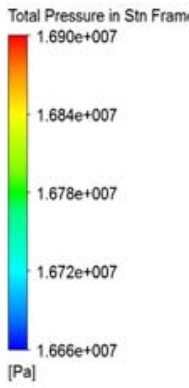

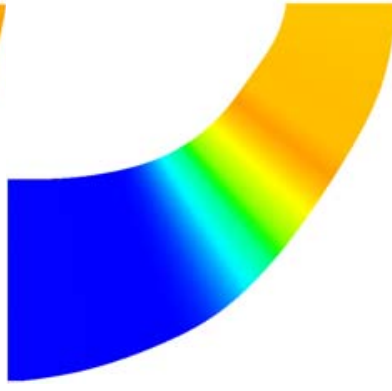

(b)

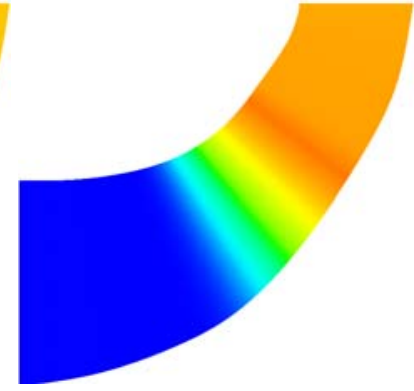

(c)

Figure 10. Total pressure distribution contour on the meridional plane of impeller and diffuser: (a) The initial model; (b) The meridian optimization; (c) The blade shape optimization

Impeller reaction factor, the ratio of potential and theoretical head of impeller, was proposed to analyze the influence of pressure energy and kinetic energy distribution on the pump efficiency and flow field of diffuser by comparing calculation results of the initial and optimum models. Table 6 shows the calculation of the impeller reaction factor. As the optimization progressed, the impeller reaction factor was gradually increased. That is, fluid with higher pressure energy in the impeller outlet is more conducive to the fluid flow in the diffuser. This was because the impeller reaction factor increased so that the potential head of impeller increased and the kinetic energy decreased. The hydraulic loss in the flow components was proportional to the nth power of average flow rate, so the hydraulic losses in the impeller and diffuser gradually reduced. The presence of whirlpool zone in the diffuser also indicated that boundary layer separation occurred in the suction surface of diffuser and the kinetic energy was not effectively converted to pressure energy, which resulted in hydraulic losses increased. The vortex region, which became smaller and was shifted after the first step optimization, indicated that absolute velocity at the impeller exit had changed, thus affecting the flow field structure in the diffuser.

Table 6. Calculation results of impeller reaction factor at the entire flow passage

\begin{tabular}{ccccc}
\hline Model & $\begin{array}{c}\text { Potential head } \\
\text { of impeller }\end{array}$ & $\begin{array}{c}\text { Total head of } \\
\text { impeller }\end{array}$ & $\begin{array}{c}\text { Impeller } \\
\text { reaction factor }\end{array}$ & $\begin{array}{c}\text { Hydraulic efficiency of } \\
\text { impeller and diffuser }\end{array}$ \\
\hline $\begin{array}{c}\text { The initial model } \\
\begin{array}{c}\text { The first meridian } \\
\text { optimization }\end{array}\end{array}$ & 18.7 & 23.1 & 0.810 & 0.911 \\
$\begin{array}{c}\text { The second blade } \\
\text { shape optimization }\end{array}$ & 16.9 & 19.5 & 0.867 & 0.930 \\
\hline
\end{tabular}

\section{Conclusion}

In this paper, a design method of pump hydraulic performance based on one-dimensional design and threedimensional optimization was proposed. The parametric models of pump impeller and diffuser were constructed. Pump hydraulic efficiency was treated as optimization objective function and the head was restriction function. The radial basis function was adopted to approximate the performance indicators. CORS-BRF optimization algorithm for global optimization was used to optimize a mixed-flow pump. After the optimization, the impact loss at the blade leading edge was reduced and the pressure distribution of diffuser was more uniform than that in the initial one under the design flow rate. Pump performance of the final optimum model improved significantly compared to the initial model. Pump head nearly unchanged, which controlled the pump input power effectively. The maximum head deviation between the predicted performance of the surrogate models and numerical results is $2.9 \%$, and the efficiency deviation is $0.92 \%$. The response surface model has high prediction accuracy. Though a little difference exists between actual situation and CFD calculation based on certain precision, this method can effectively improve the pump performance. What's more, using the single passage for optimization is feasible which results in reducing large amount of computing time. The calculation errors between the single passage and entire passage can't affect the overall optimization trend. The optimization results also show that the energy distribution of impeller exit has an important effect on the flow state of diffuser. The conversion of more fluid energy to pressure energy is more conducive to reducing the flow loss in the diffuser. In addition, the proposed two-step optimization method of the meridional plane and blade shape can effectively solve the optimization problem of large numbers of parameters, 
and has a good prospect. However, in the case that the classification of design parameters is not clear. The study on design parameters of each sub-optimization process interacting with each other should also be continued.

\section{Acknowledgments}

This work was supported by National Natural Science Foundation of China (11402047), National Program on Key Basic Research Project (2015CB057301), Fundamental Research Funds for the Central Universities (DUT17GF202) and Collaborative Innovation Center of Major Machine Manufacturing in Liaoning.

\section{Reference}

1. Škerlavaj, A.; Morgut M., Jošt, D. Optimization of a single-stage double-suction centrifugal pump. Journal of Physics Conference Series. Journal of Physics Conference Series, 2017: 012007.

2. Huang, R.F.; Luo, X.W.; Ji, B. Multi-objective optimization of a mixed-flow pump impeller using modified NSGA-II algorithm. Science China Technological Sciences, 2015, 58: 2122-2130.

3. Yuan, S.; Wang, W.; Pei, J. Multi-objective optimization of low-specific-speed centrifugal pump. Transactions of the Chinese Society of Agricultural Engineering (Transactions of the CSAE), 2015, 31(5): 46-52.

4. Xu, S.L.; Liu, H.T.; Wang, X.F. Mass optimization of turbine disk based on sequential sampling algorithm. Journal of Aerospace Power, 2014, 29(9): 2098-2102.

5. Bing, H. Study on the design theory and optimization method of mixed flow pump impeller with diffuser vanes. Dissertation for the Doctor Degree. Beijing: Tsinghua University, 2012.

6. Tan, L. Study on theory and method of optimization design for centrifugal pump impeller. Dissertation for the Doctor Degree. Beijing: Tsinghua University, 2011.

7. Regis, R.G.; Shoemaker, C.A. Constrained Global Optimization of Expensive Black Box Functions Using Radial Basis Functions. Journal of Global Optimization, 2005, 31: 153-171.

8. Lai, X.D.; Chen, X.M.; Zhang, X. An Approach to automatically optimize the Hydraulic performance of Blade System for Hydraulic Machines using Multi-objective Genetic Algorithm IOP Conference Series: Earth and Environmental Science. IOP Conference Series: Earth and Environmental Science, 2016: 062027.

9. Heo, M.W.; Ma, S.B.; Shim, H.S. High-efficiency design optimization of a centrifugal pump. Journal of Mechanical Science \& Technology, 2016, 30(9): 3917-3927.

10. Kim, S.; Lee, K.Y.; Kim, J.H. High performance hydraulic design techniques of mixed-flow pump impeller and diffuser. Journal of Mechanical Science and Technology, 2015, 29(1):227-240.

11. Casey, M.V. A computational geometry for the blades and internal flow channels of centrifugal compressors. Transaction of the ASME, Journal of Engineering for Power, 1983, 105:288-295.

12. Braembussche, V.D. and R. A. Challenges and progress in turbomachinery design systems. Materials Science and Engineering 52 (2013) 012001. 